October 2, 2018

\title{
Phase transitions in $\mathrm{BaTiO}_{3}$ from first principles
}

\author{
W. Zhong and David Vanderbilt \\ Department of Physics and Astronomy, Rutgers University, Piscataway, NJ 08855-0849 \\ K. M. Rabe \\ Department of Applied Physics, Yale University, New Haven, CT 06520
}

(October 2, 2018)

\begin{abstract}
We develop a first-principles scheme to study ferroelectric phase transitions for perovskite compounds. We obtain an effective Hamiltonian which is fully specified by first-principles ultra-soft pseudopotential calculations. This approach is applied to $\mathrm{BaTiO}_{3}$, and the resulting Hamiltonian is studied using Monte Carlo simulations. The calculated phase sequence, transition temperatures, latent heats, and spontaneous polarizations are all in good agreement with experiment. The order-disorder vs. displacive character of the transitions and the roles played by different interactions are discussed.
\end{abstract}

Submitted to Phys. Rev. Lett.

77.80.Bh, 61.50.Lt, 64.60.Cn, 64.70.-p 
Because of their simple crystal structure, the cubic ferroelectric perovskites present a special opportunity for the development of a detailed theoretical understanding of the ferroelectric phase transition. However, even in $\mathrm{BaTiO}_{3}$, a much-studied prototypical example of this class of compounds [1], many aspects of the phase behavior are far from simple. $\mathrm{BaTiO}_{3}$ undergoes a succession of phase transitions, from the high-temperature high-symmetry cubic perovskite phase (Fig. 1) to slightly distorted ferroelectric structures with tetragonal, orthorhombic and rhombohedral symmetry. There is increasing evidence that the cubictetragonal transition, at first thought to be of the simple displacive kind, may instead be better described as of the order-disorder type.

A comparison with the related cubic perovskites indicates that this and other aspects of the phase transformation behavior in $\mathrm{BaTiO}_{3}$ are not universal, but rather must depend on details of the chemistry and structural energetics of the particular compound. Therefore, it is of the first importance to develop a microscopic theory of the materials properties which determine the ordering of the phases, the character and thermodynamic order of the transitions, and the transition temperatures. The value of a microscopic approach has long been appreciated, but its realization was hindered by the difficulty of determining microscopic parameters for individual compounds. The forms of phenomenological model Hamiltonians [1] [biguities in interpretation. For the perovskite oxides, empirical [5] and nonempirical pair potential methods [6] did not offer the high accuracy needed for the construction of realistic models. Recently, high quality first-principles calculations within the local density approximation (LDA) have been shown to provide accurate total-energy surfaces for perovskites [7[10]. While an ab-initio molecular-dynamics simulation of the structural phase transition is not computationally feasible at present, the application of these first-principles methods can clearly form a foundation for the realistic study of the finite-temperature phase transitions.

In this paper, we pursue a completely first-principles approach to study the ferroelectric phase transitions in $\mathrm{BaTiO}_{3}$. In particular, we (i) construct an effective Hamiltonian to 
describe the important degrees of freedom of the system [11, 12], (ii) determine all the parameters of this effective Hamiltonian from high-accuracy ab-initio LDA calculations [9, 13, 14,, and (iii) carry out Monte Carlo (MC) simulations to determine the phase transformation behavior of the resulting system. We find the correct succession of phases, with transition temperatures and spontaneous polarization in reasonable agreement with experiment. Strain coupling is found to be crucial in producing the correct succession of low-symmetry phases. Finally, by analyzing the local distortions and phonon softening, we find the cubic-tetragonal transition in $\mathrm{BaTiO}_{3}$ to be intermediate between the displacive and order-disorder limits.

Briefly, the effective Hamiltonian is constructed as follows. Since the ferroelectric transition involves only small structural distortions, we represent the energy surface by a Taylor expansion around the high-symmetry cubic perovskite structure, including fourth-order anharmonic terms. Because the contribution to the partition function decays exponentially with increasing energy, we simplify this expansion by including only low energy distortions. Among all the possible phonon excitations, the long-wavelength acoustic modes (strain) and lowest transverse-optical phonon modes (soft modes) have the lowest energy. It is therefore our approximation to include only these two kinds of phonon excitations, thus reducing the number of degrees of freedom per unit cell from fifteen to six. This approximation could later be systematically improved, or entirely removed, by including higher-energy phonons.

It is straightforward to describe the strain degrees of freedom associated with the acoustic modes in terms of displacement vectors $\mathbf{v}_{l}$ associated with each unit cell $l$. In a similar manner, we introduce variables $\mathbf{u}_{l}$ to describe the amplitude of a "local mode" associated with cell $l$. The properly chosen local mode should reproduce the soft-mode phonon dispersion relation throughout the Brillouin zone, preserve the symmetry of the crystal, and minimize interactions between adjacent local modes. The local mode chosen for $\mathrm{BaTiO}_{3}$ is shown in Fig. 1. The terms in our Taylor expansion of the energy in the variables $\{\mathbf{u}\}$ and $\{\mathbf{v}\}$ are organized as follows: (i) a soft-mode self-energy $E^{\text {self }}(\{\mathbf{u}\})$ containing intrasite interactions to quartic anharmonic order; (ii) a long-range dipole-dipole coupling $E^{\mathrm{dpl}}(\{\mathbf{u}\})$ and a short-range (up to third neighbor) correction $E^{\text {short }}(\{\mathbf{u}\})$ to the intersite coupling, 
both at harmonic order; (iii) a harmonic elastic energy $E^{\text {elas }}(\{\mathbf{v}\})$; and (iv) an anharmonic strain-soft-mode coupling $E^{\text {int }}(\{\mathbf{u}\},\{\mathbf{v}\})$ containing Gruneisen-type interactions (i.e., linear in strain and quadratic in soft-mode variables). The cubic symmetry greatly reduces the number of expansion coefficients needed. All the expansion parameters are determined from highly-accurate first-principles LDA calculations applied to supercells containing up to four primitive cells (20 atoms). The calculation of the needed microscopic parameters within LDA for $\mathrm{BaTiO}_{3}$ has been made possible by the use of Vanderbilt ultra-soft pseudopotentials [13, which make large-scale calculations tractable at the high level of accuracy needed, and by the recent theory of polarization of King-Smith and Vanderbilt [15], which provides a convenient method of calculating the dipolar interaction strengths [14]. The details of the Hamiltonian, the first-principles calculations, and the values of the expansion parameters will be reported elsewhere [16].

We solve the Hamiltonian using Metropolis Monte Carlo simulations [17, 18] on an $L \times$ $L \times L$ cubic lattice with periodic boundary conditions. The homogeneous part of the strain in the system is separated out and treated as six extra degrees of freedom. Since most energy contributions (except $E^{\mathrm{dpl}}$ ) are local, we choose the single-flip algorithm and define one Monte Carlo sweep (MCS) as $L^{3}$ flip attempts.

The ferroelectric phase transition is very sensitive to hydrostatic pressure, or equivalently, to lattice constant. The LDA-calculated lattice constants are typically $1 \%$ too small, and even this small error can lead to large errors in the zero-pressure transition temperatures. The effect of this systematic error can largely be compensated by exerting a negative pressure that expands the lattice constant to the experimental value. For $\mathrm{BaTiO}_{3}$, we choose $P=$ -4.8 $\mathrm{GPa}$ which gives the best overall agreement for the computed volumes for the four phases with their experimental values. The following simulations and analysis are for this pressure.

In our simulation, we concentrate on identifying the succession of different phases, determining the phase transition temperatures, and extracting qualitative features of the transitions. We also focus on identifying the features of the Hamiltonian which most strongly 
affect the transition properties. For these purposes, it is most convenient to monitor directly the behavior of the order parameter. In the case of the ferroelectric phase transition, this is just the polarization vector (or equivalently, the soft-mode amplitude vector $\mathbf{u}$ ) averaged over the simulation cell. To avoid effects of possible rotation of the polarization vector and to identify the different phases clearly, we choose to accumulate the absolute values of the largest, middle, and smallest components of the averaged local-mode vector for each step, denoted by $u_{1}, u_{2}$, and $u_{3}$, respectively $\left(u_{1}>u_{2}>u_{3}\right)$. The cubic (C), tetragonal (T), orthorhombic $(\mathrm{O})$, and rhombohedral $(\mathrm{R})$ phases are then characterized by zero, one, two, and three non-zero order-parameter components, respectively. As a reference, the average local mode amplitude $u=\sum_{i}\left|\mathbf{u}_{i}\right| / N$ is also monitored. Here, $\mathbf{u}_{i}$ is the local mode vector at site $i$ and $N$ is the total number of sites.

Fig. 2 shows the quantities $u_{1}, u_{2}, u_{3}$, and $u$ as functions of temperature in a typical simulation for an $L=12$ lattice. For clarity, we show only the cooling down process. The values are averaged over 7000 MCS's after the system reaches equilibrium, so that the typical fluctuation of order parameter components is less than $10 \%$. We find that $u_{1}, u_{2}$, and $u_{3}$ are all very close to zero at high temperature. As the system cools down past 340K, $u_{1}$ increases and becomes significantly larger than $u_{2}$ or $u_{3}$. This indicates the transition to the tetragonal phase. The homogeneous-strain variables confirm that the shape of the simulation cell becomes tetragonal. Two other phase transitions occur as the temperature is reduced further. The $\mathrm{T}-\mathrm{O}$ transition occurs at $255 \mathrm{~K}$ (sudden increase of $u_{2}$ ) and the $\mathrm{O}-\mathrm{R}$ transition occurs at $210 \mathrm{~K}$ (sudden increase of $u_{3}$ ). The shape of the simulation cell also shows the expected changes. The sequence of transitions exhibited by the simulation is the same as that observed experimentally.

The transition temperatures are located by careful cooling and heating sequences. We start our simulation at a high temperature and equilibrate in the cubic phase. The temperature is then reduced in small steps. At each temperature, the system is allowed to relax for 10,000 MCS (increased to 25,000 and then to 40,000 MCS's close to the transition). After each transition is complete, the system is reheated slowly to detect any possible hysteresis. 
The calculated transition temperatures are shown in Table [. Simulations for three lattice sizes are performed; the error estimates in the table reflect the hysteretic difference between cooling and heating, which persists even after significant increase of the simulation time. (The $\mathrm{C}-\mathrm{T}$ transition temperature for $L=10$ is difficult to identify because of large fluctuations between phases.) The calculated transition temperatures are well converged with respect to system size, and are in good agreement with experiment. The saturated spontaneous polarization $P_{\mathrm{s}}$ in different phases can be calculated from the average local mode

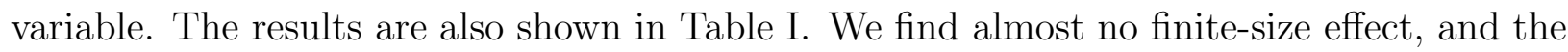
agreement with experiment is very good for $\mathrm{O}$ and $\mathrm{T}$ phases. The disagreement for the $\mathrm{R}$ phase may be due in part to twinning effects in the experimental sample [19].

One way to determine the order of the transition is to calculate the latent heat. An accurate determination of the latent heat would require considerable effort; here, we only try to provide good estimates. We approach the transition from both high-temperature and low-temperature sides until the point is reached where both phases appear equally stable. The difference of the average total energy is then the latent heat [20]. This estimate should

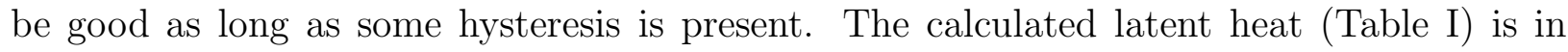
rough agreement with the rather scattered experiment data. The discrepancy for the $\mathrm{C}$ $\mathrm{T}$ transition can be partly attributed to the finite-size effect. We find that, taking into account finite-size effects, the latent heats for all three transitions are significantly non-zero, suggesting all transitions are first-order. For the $\mathrm{T}-\mathrm{O}$ and $\mathrm{O}-\mathrm{R}$ transitions, this is consistent with Landau theory, which requires a transition to be first-order when the subgroup relation does not hold between the symmetry groups below and above $T_{c}$. For the $\mathrm{C}-\mathrm{T}$ transition, although it has the largest latent heat, we find indications that it is the most weakly first order. The relatively large finite-size effect suggests a relatively long correlation length near the transition, and the change of the order parameter during the transition is also more gradual (Fig.2).

Next, we investigate the extent to which the cubic-tetragonal transition can be characterized as order-disorder or displacive. In real space, these possibilities can be distinguished 
by inspecting the distribution of the local-mode vector $\mathbf{u}_{i}$ in the cubic phase just above the transition. A displacive (microscopically nonpolar) or order-disorder (microscopically polar) transition should be characterized by a single-peak or double-peak structure, respectively. The distribution of $u_{x}$ at $T=400 \mathrm{~K}$ is shown in Fig. 3 . It exhibits a rather weak tendency to a double-peaked structure, indicating a transition which has some degree of order-disorder character. We also see indications of this in the $u-T$ relation in Fig.2; even in the cubic phase, the magnitude of the local-mode vector $u$ is significantly non-zero and close to that of the rhombohedral phase. Although the components of the local modes change dramatically during the phase transition, $u$ only changes slightly.

In reciprocal space, a system close to a displacive transition should show large and strongly temperature-dependent fluctuations of certain phonons (soft modes) confined to a small portion of the Brillouin zone (BZ). For an extreme order-disorder transition, on the other hand, one expects the fluctuations to be distributed over the whole BZ. For $\mathrm{BaTiO}_{3}$, we calculated the average Fourier modulus of the soft TO mode $\left\langle|u(q)|^{2}>\right.$ at several temperatures just above the $\mathrm{C}-\mathrm{T}$ transition. A strong increase of $\left\langle|u(q)|^{2}>\right.$ as $T \rightarrow T_{c}$ would indicate phonon softening. As expected, we do observe this behavior for modes at $\Gamma$. While these modes become "hard" rather quickly along most directions away from $\Gamma$, they remain soft at least half-way to the BZ boundaries along the $\{100\}$ directions, again indicating some order-disorder character. Thus, from the example of $\mathrm{BaTiO}_{3}$, it seems that a positive on-site quadratic coefficient does not automatically imply a displacive character for the transition. Rather, the relevant criterion is the extent to which the unstable phonons extend throughout the BZ.

Our theoretical approach allows us to investigate the roles played by different types of interaction in the phase transition. First, we study the effect of strain. Recall that the strain degrees of freedom were separated into local and homogeneous parts, representing finite- and infinite-wavelength acoustic modes, respectively. Both parts were included in the simulations. If we eliminate the local strain (while still allowing homogeneous strain), we find almost no change in the transition temperatures. This indicates that the effect of the 
short-wavelength acoustic modes may not be important for the ferroelectric phase transition. If the homogeneous strain is frozen, however, we find a direct cubic-rhombohedral phase transition, instead of the correct series of three transitions. This demonstrates the important role of homogeneous strain. Second, we studied the significance of the long-range Coulomb interaction in the simulation. To do this, we changed the effective charge of the local mode (and thus the dipole-dipole interaction), while modifying other parameters so that the frequencies of the zone-center and zone-boundary phonons remain in agreement with the LDA values. We found only a slight change (10\%) of the transition temperatures when the dipole-dipole interaction strength was reduced by half, but a further reduction changed the results dramatically (in fact the ground state becomes a complex antiferroelectric structure). This result shows that it is essential to include the long-range interaction, although small inaccuracies in the calculated values of the effective charges or dielectric constants may not be very critical. On the other hand, our tests do indicate a strong sensitivity of the $T_{c}$ 's to any deviation of the fitted zone-center or zone-boundary phonon frequencies away from the LDA results. Thus, highly accurate LDA calculations do appear to be a prerequisite for an accurate determination of the transition temperatures.

Our approach opens several avenues for future study. Allowing a higher-order expansion of the energy surface might allow an accurate determination of the phase diagram. More extensive Monte-Carlo simulations on larger systems, and with careful analysis of finite-size scaling, could provide more precise transition temperatures, free energies, and latent heats [21]. Finally, the theory would be more satisfying if the $1 \%$ underestimate of the lattice constant in the LDA calculation could be reduced or eliminated.

In conclusion, we have developed a first-principles approach to the study of structural phase transitions and the calculation of transition temperatures in $\mathrm{BaTiO}_{3}$. We have obtained the transition sequence, transition temperatures, and spontaneous polarizations, and found them to be in good agreement with experiment. We find that long-wavelength acoustic modes and long-range dipolar interactions both play an important role in the phase transition, while short-wavelength acoustic modes are not as relevant. The $\mathrm{C}-\mathrm{T}$ phase tran- 
sition is not found to be well described as a simple displacive transition; on the contrary, if anything it has more order-disorder character.

We would like to thank R.D. King-Smith, U. V. Waghmare, R. Resta, Z. Cai, and A.M. Ferrenberg for useful discussions. This work was supported by the Office of Naval Research under contract numbers N00014-91-J-1184 and N00014-91-J-1247. 


\section{REFERENCES}

[1] M. E. Lines and A. M. Glass, Principles and Applications of Ferroelectrics and Related Materials, (Clarendon Press, Oxford, 1977).

[2] M. T. Dove, A. P. Giddy, and V. Heine, Ferroelectrics 136, 33 (1992).

[3] E. Pytte, Phys. Rev. B 5, 3758 (1972).

[4] A. D. Bruce and R. A. Cowley, Structural Phase Transitions, (Taylor \& Francis, London, 1981).

[5] H. Bilz, G. Benedek, and A. Bussmann-Holder, Phys. Rev. B 87, 4840 (1987) and references therein.

[6] G. Gordon and Y. S. Kim, J. Chem. Phys. 56, 3122 (1972); L. L. Boyer et al, Phys. Rev. Lett. 54, 1940 (1985); P. J. Edwardson et al, Phys. Rev. B 39, 9738 (1989).

[7] R. E. Cohen and H. Krakauer, Phys. Rev. B 42, 6416 (1990); R. E. Cohen and H. Krakauer, Ferroelectrics 136, 65 (1992); R. E. Cohen, Nature 358, 136 (1992).

[8] D. J. Singh and L. L. Boyer, Ferroelectrics 136, 95 (1992).

[9] R. D. King-Smith and D. Vanderbilt, Ferroelectrics 136, 85 (1992).

[10] R. D. King-Smith and D. Vanderbilt, Phys. Rev. B 49, 5828 (1994).

[11] K. M. Rabe and J. D. Joannopoulos, Phys. Rev. Lett. 59, 570 (1987); Phys. Rev. B 36, 6631 (1987).

[12] K. M. Rabe and U. V. Waghmare, to be published in Ferroelectrics.

[13] D. Vanderbilt, Phys. Rev. B 41, 7892 (1990).

[14] W. Zhong, R. D. King-Smith and D. Vanderbilt, Phys. Rev. Lett. 72, 3618 (1994).

[15] R. D. King-Smith and D. Vanderbilt, Phys. Rev. B 47, 1651 (1993); D. Vanderbilt and R. D. King-Smith, Phys. Rev. B 48, 4442 (1993). 
[16] W. Zhong, D. Vanderbilt, and K. M. Rabe (unpublished).

[17] N. Metropolis et al, J. Chem. Phys. 21, 1087 (1953).

[18] M. P. Allen and D. J. Tildesley, Computer Simulation of Liquids (Oxford, New York, 1990); K. Binder and D. W. Heermann, Monte Carlo Simulation in Statistical Physics (Springer-Verlag, Berlin, 1988); K. Binder, ed. The Monte Carlo Method in Condensed Matter Physics, (Springer-Verlag, Berlin, 1992).

[19] H. H. Wieder, Phys. Rev. 99, 1161 (1955).

[20] Under a physical applied pressure, the latent heat would be the change of enthalpy, not energy. If this were applied to our case of a fictitious pressure, the calculated latent heats would be approximately twice as large.

[21] J. Lee and J. M. Kosterlitz, Phys. Rev. Lett. 65, 137 (1990); Phys. Rev. B 43, 3265 (1991); A. M. Ferrenberg and R. H. Swendsen, Phys. Rev. Lett. 61, 2635 (1988); 63, 1195 (1989). 


\section{TABLES}

TABLE I. Calculated transition temperatures $T_{\mathrm{c}}$, saturated spontaneous polarization $\mathrm{P}_{\mathrm{s}}$, and estimated latent heat $l$, as a function of simulation cell size.

\begin{tabular}{|c|c|c|c|c|c|}
\hline & phase & $L=10$ & $L=12$ & $L=14$ & $\operatorname{expt}^{\mathrm{a}}$ \\
\hline \multirow{3}{*}{$T_{\mathrm{c}}(\mathrm{K})$} & $\mathrm{O}-\mathrm{R}$ & $210 \pm 10$ & $220 \pm 10$ & $218 \pm 2$ & 183 \\
\hline & $\mathrm{T}-\mathrm{O}$ & $252 \pm 2$ & $260 \pm 5$ & $264 \pm 1$ & 278 \\
\hline & $\mathrm{C}-\mathrm{T}$ & $\sim 320$ & $341 \pm 1$ & $342 \pm 2$ & 403 \\
\hline \multirow{3}{*}{$\mathrm{P}_{\mathrm{s}}\left(\mathrm{C} / \mathrm{m}^{2}\right)$} & $\mathrm{R}$ & 0.43 & 0.43 & 0.43 & 0.33 \\
\hline & $\mathrm{O}$ & 0.36 & 0.35 & 0.36 & 0.36 \\
\hline & $\mathrm{T}$ & 0.28 & 0.28 & 0.28 & 0.27 \\
\hline \multirow{3}{*}{$l(\mathrm{~J} / \mathrm{mol})$} & $\mathrm{O}-\mathrm{R}$ & 58 & 50 & 50 & $33-60$ \\
\hline & $\mathrm{T}-\mathrm{O}$ & $\geq 86$ & 92 & 100 & 65-92 \\
\hline & $\mathrm{C}-\mathrm{T}$ & - & $\geq 73$ & 115 & 196-209 \\
\hline
\end{tabular}

aT. Mitsui et al., Landolt-Bornstein numerical data and functional relationships in science and technology (Springer-Verlag, 1981), NS, III/16. 


\section{FIGURES}

FIG. 1. The structure of cubic perovskite compounds $\mathrm{BaTiO}_{3}$. Atoms $\mathrm{Ba}$, Ti and $\mathrm{O}$ are represented by shaded, solid, and empty circles respectively. The areas of the vectors indicate the magnitudes of the displacements for a local mode polarized along $\hat{\mathbf{x}}$.

FIG. 2. The averaged largest, middle, and smallest components $u_{1}, u_{2}, u_{3}$ and amplitude $u$ of local modes as a function of temperature in a cooling-down simulation of a $12 \times 12 \times 12$ lattice. The dotted lines are guides to the eyes.

FIG. 3. The distribution of a Cartesian component of the local mode variable in the cubic phase at $T=400 K$. 